\title{
$\mathrm{Ka}$-대역 $\mathrm{QFN}$ 패키지의 본딩 와이어 분석 및 설계
}

\section{Design and Analysis of Bonding Wire for Ka-Band QFN Package}

\author{
왕 문 걸.강 원 실* \\ Wenjie Wang · Wonshil Kang* · Hyunchul Ku
}

요 약

본 논문에서는 Ka-대역 QFN(quad flat no-lead) 패키지에 사용되는 와이어 본딩 기법에서의 본딩 와이어의 전기적 특성 을 분석하여 등가 모델을 제안하고, 그 유효성을 검증하였다. 본딩 와이어의 길이에 따른 인덕턴스와 저항 성분 변화 및 다중 본딩 와이어에서 발생하는 상호 인덕턴스를 고려하여 등가 모델을 개발하였다. 등가 모델의 각 소자 성분 값을 유도하기 위한 해석적 방법을 제시하고, 그 모델의 유효성을 검증하기 위하여 모델에서 유도된 $S$-파라미터 결과를 3차원 전자기 시뮬레이션 결과와 비교 제시하였다. 제안된 본딩 와이어 등가 모델을 이용하여 설계된 Ka-대역 $\mathrm{QFN}$ 패키지에서 반사 손실과 삽입 손실을 최적화한 본딩 와이어의 개수 및 길이를 도출하고, 이를 적용하여 $\mathrm{QFN}$ 패키지를 제작하고 $S$-파라미터 성능을 검증하였다. 설계 제작된 $\mathrm{Ka}$-대역 $\mathrm{QFN}$ 패키지 본딩 와이어의 $\mathrm{RF}$ 특성이 $29 \mathrm{GHz}$ 에서 $S_{11}$ (반사 손실)이 $-26 \mathrm{~dB}, S_{21}$ (삽입 손실)이 $-1.8 \mathrm{~dB}$ 의 성능을 보였으며, 모의실험 결과와 유사한 결과임을 확인하였다.

\begin{abstract}
In this study, an equivalent model of bonding wire used in the Ka-band quad flat no-lead (QFN) package was proposed. The proposed equivalent model was constructed using resistors and inductors according to the length of the bonding wire and the mutual inductance between the multi-bonding wires. For verification, the $S$-parameters derived from the equivalent model were compared with the three-dimensional (3D) electromagnetic simulation results. Moreover, a Ka-band QFN package was designed using the proposed equivalent model. The number and length of the bonding wires required to optimize the return loss $\left(S_{11}\right)$ and the insertion loss $\left(S_{21}\right)$ of the designed Ka-band QFN package were derived and applied. In the fabricated Ka-band QFN package, the $S_{11}$ and $S_{21}$ were measured to be -26 $\mathrm{dB}$ to $-1.8 \mathrm{~dB}$ at $29 \mathrm{GHz}$, respectively, and it was confirmed that the measurement results were similar to the simulation results.
\end{abstract}

Key words: 5G, Equivalent Model, Ka-Band, QFN(Quad Flat No-Lead Package), Wire Bonding

\section{I. 서 론}

최근 상용화된 $5 \mathrm{G}$ 이동통신은 고속 데이터 통신 지원
을 위해 새로운 주파수 대역에서 더 넓은 대역폭을 필요 로 한다. 국내 이동통신 3 사는 $5 \mathrm{G}$ 서비스를 위해 $3.5 \mathrm{GHz}$ 주변 $S$-대역에서 $280 \mathrm{MHz}$ 와 $28 \mathrm{GHz}$ 주변 $\mathrm{Ka}$-대역에서

\footnotetext{
「이 연구는 산업통상자원부(과제 번호: 20004102) 및 한국산업기술평가관리원(KEIT)과 정부(과학기술정보통신부)의 재원으로 한국연구재단의 지원 을 받아 수행된 연구임(NRF-2020R1F1A1061826).」 건국대학교 전자정보통신공학과(Department of Electronic Engineering, Konkuk University) *알에프머트리얼즈(RF Materials)

• Manuscript received September 30, 2021 ; Revised October 13, 2021 ; Accepted November 24, 2021. (ID No. 20210930-083)

· Corresponding Author: Hyunchul Ku (e-mail: hcku@konkuk.ac.kr)
} 
THE JOURNAL OF KOREAN INSTITUTE OF ELECTROMAGNETIC ENGINEERING AND SCIENCE. vol. 32, no. 11, November. 2021.

$2,400 \mathrm{MHz}$ 의 주파수 대역폭을 2018년에 할당받았으며, 2019년부터 $S$-대역을 중심으로 $5 \mathrm{G}$ 서비스를 제공하고 있 다. $5 \mathrm{G}$ 시스템을 활용하여 초광대역 고속 데이터 전송을 위해서는 $S$-대역뿐만 아니라, Ka-대역의 적극적인 활용이 필요하다. 이를 위해서는 경제적이고 성능이 우수한 $\mathrm{Ka}-$ 대 역용 $\mathrm{RF}$ (radio frequency) 반도체 부품의 개발이 필요하다. 반도체 패키지 중 QFN(quad flat no-lead) 패키지는 4개 의 방향으로 연결되는 Lead-frame과 패키지 아랫면에 패 드를 배치하여 집적도를 높이고 실장면적을 줄인 구조로 서 소형화, 저비용, 고성능이 가능하여 RF 부품의 패키지 에 다양하게 활용되고 있다. QFN 패키지 내의 Lead-frame 패드와 $\mathrm{MMIC}$ (monolithic microwave integrate circuits) 다이 의 패드를 연결하기 위해 와이어 본딩(wire bonding) 기법, 플립-칩 본딩(flip-chip bonding) 기법, $\mathrm{TAB}$ (tape automated bonding) 기법이 있다. 패키지와 MMIC 칩의 접속 기술은 고주파 대역에서 $\mathrm{QFN}$ 패키지의 시스템 전체 성능에 영 향을 미치는 핵심적인 요소라고 할 수 있다[1],[2]. 다양한 기법 중 와이어 본딩 방식이 가장 일반적으로 사용되고 있으며, 다양한 재료의 와이어에 대한 성능 검증 및 연구 가 활발히 진행되고 있다 ${ }^{[3]-[5]}$.

반도체 패키지의 본딩 와이어는 낮은 주파수 대역에서 이상적인 전송선로로 동작하지만, 전송 신호의 주파수가 증가함에 따라 기생 리액턴스 성분이 증가하게 되어 $\mathrm{RF}$ 신호의 전송 특성에 큰 영향을 미치게 된다. 따라서 $\mathrm{Ka}-$ 대역 $\mathrm{QFN}$ 패키지 기반의 $5 \mathrm{G} \mathrm{RF}$ 부품 개발을 위해서는 $\mathrm{QFN}$ 패키지 내의 본딩 와이어의 전기적 특성을 정확하 게 분석하여 모델링하고, 이를 기반으로 원하는 RF 특성 을 얻을 수 있는 본딩 와이어 설계 기법에 대한 연구가 필요하다.

본딩 와이어의 특성 분석 및 설계를 위해 참고문헌 [5] 에서는 주파수 종속 저항 $\left(R_{s k i n} f^{m}\right)$ 을 사용하여 더블- $\pi$ 형 본딩 와이어 모델을 제시하고, 두 종류의 물질(알루미늄/ 금)에 대한 와이어 길이의 변화에 따른 연구를 진행하였 다. 추출된 본딩 와이어의 등가회로 모델의 파라미터를 표시하고, $S$-파라미터를 통해서 본딩 와이어의 R, L, C 기 생성분을 검증하였다. 또한, 참고문헌 [6]에서는 본딩 와 이어의 $\mathrm{EM}$ 구조 모델을 이용해 와이어의 지름, 높이 등 을 설계하고, $\mathrm{RF}$ 회로 이론 기반으로 시뮬레이션한 $S$-파
라미터를 통해서 등가회로 모델을 제시하고 검증하였다. 참고문헌 [7]에서는 칩과 패키지 사이의 다양한 본딩 와 이어에서 와이어의 길이를 변화와 임피던스 부정합이 발 생할 때 RF 특성을 연구하였다. 하지만, 위의 연구들은 $\mathrm{K}$-대역 이하 주파수에 대한 연구로, $5 \mathrm{G}$ 이동통신 주파수 인 Ka-대역에 적합하지 않고, 패키지의 구조를 포함한 분 석을 진행하지 않았다.

따라서 본 논문에서는 $\mathrm{Ka}$-대역에서 본딩 와이어의 길 이, 개수에 따른 와이어간 상호 인덕턴스를 고려한 등가 모델을 제안하고, 3 차원 $\mathrm{EM}$ 시뮬레이션을 통해서 본딩 와이어의 인덕턴스 및 결합계수를 예측하고, 이를 도출한 본딩 와이어를 설계하였다. 설계된 본딩 와이어를 $7 \times 7$ $\mathrm{mm}$ 24-Pin QFN 패키지에 적용하여 제작하고, Ka-대역에 서의 $S$-파라미터 특성을 검증하였다. 본 논문의 구성은 다음과 같다.

2장에서는 $\mathrm{QFN}$ 패키지 본딩 와이어의 모델링 방법을 제시하고, 3장에서는 $\mathrm{Ka}$-대역용 $\mathrm{QFN}$ 패키지에 적용하기 위한 본딩 와이어를 설계하고 모의실험을 진행하였다. 4 장에서는 $\mathrm{QFN}$ 패키지를 제작하고 실험 및 결과를 제시 하고, 5 장에서는 결론을 맺는다.

\section{QFN 패키지의 본딩 와이어 모델링}

본딩 와이어의 저항, 커패시턴스 및 인덕턴스의 기생 성분들은 주파수가 증가함에 따라 skin effect로 인해 지속 적으로 변화한다 ${ }^{[8]}$. 본 논문에서는 $\mathrm{Ka}$-대역에서 본딩 와 이어의 길이, 개수에 따른 와이어간 상호 인덕턴스를 고 려한 등가 모델을 제안하고, 3 차원 $\mathrm{EM}$ 시뮬레이션을 통 해서 본딩 와이어의 인덕턴스 및 결합계수를 예측하고, 이를 도출한 본딩 와이어를 설계하였다.

$\mathrm{QFN}$ 패키지에서 본딩 와이어에 의한 특성을 분석하기 위하여 그림 1 과 같이 $\mathrm{MMIC}$ 와 패키지의 Lead-frame을 와 이어로 연결하는 구조를 고려한다.

그림 1 에서의 본딩 와이어에 사용되는 금 와이어를 분 석하기 위해 단일 와이어의 기하학적 구조를 그림 2에 도 시하였다.

그림 2에서는 Lead-frame과 MMIC 서브스트레이트 간 의 세 개의 부분으로 구성된 단일 본딩 와이어의 구조를 


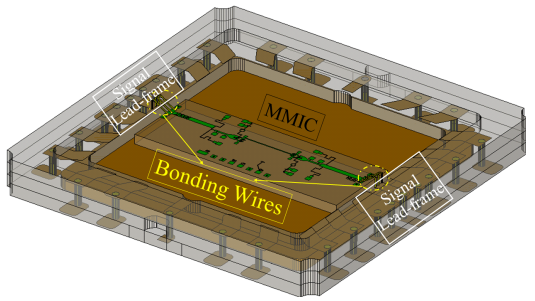

그림 1. $\mathrm{QFN}$ 패키지의 본딩 와이어 설계

Fig. 1. Bonding wire design of QFN package.

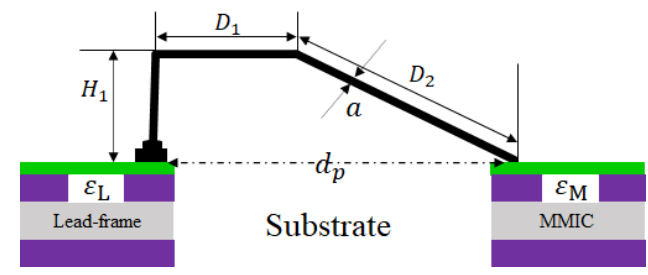

그림 2. 단일 본딩 와이어의 기하학적 구조

Fig. 2. Geometric structure of single bonding wire.

제시한다. $a$ 는 와이어의 직경, $H_{1}$ 은 와이어의 높이, $d_{p}$ 는 두 서브스트레이트 간의 수평거리, $\varepsilon_{L}, \varepsilon_{M}$ 은 두 서브스트 레이트의 유전율이다. 그림 2의 단일 본딩 와이어는 저항 과 직렬로 연결된 집중 인덕턴스의 등가회로를 그림 3 과 같이 도시할 수 있다.

그림 3에서 본딩 와이어는 고 충실도 모델(high fidelity model) 기법을 통해서 전송선로처럼 계산할 수 있다 ${ }^{[9]}$. 이 기법을 적용한 본딩 와이어의 유전체에 대한 임피던스와 와이어의 유효 유전 상수를 $Z_{i m}, x_{e f f}$ 로 표시하였다. 그림 3 에서 본딩 와이어의 자체 인덕턴스는 $L_{w}$, 저항은 $R_{w}$ 로 표시한다.

$$
L_{w}=\frac{Z_{i m} \sqrt{\varkappa_{e f f}}}{v_{0}}(H)
$$

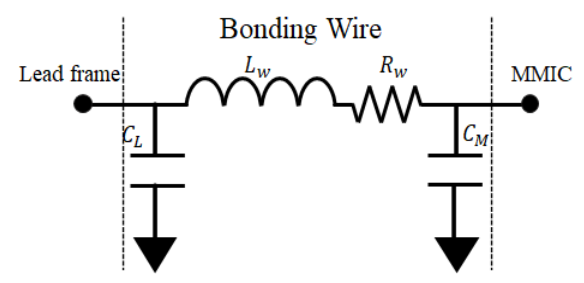

그림 3. 단일 본딩 와이어의 등가회로

Fig. 3. Equivalent circuit of single bonding wire

$$
R_{w}=\frac{D_{L}}{\sigma A}
$$

본딩 와이어는 $\left(H_{1}, D_{1}, D_{2}\right)$ 세 부분으로 구성되고, 와 이어 길이는 $D_{L}$ 로 표시한다. 진공에서 빛의 속도는 $v_{0}$ 로 표시되고 도전율은 $\sigma$, 단면 면적은 $A$ 으로 제시되었다. $C_{L}, C_{M}$ 은 와이어와 서브스트레이트 사이에서 발생된 커 패시턴스이다. Lead-frame과 $\mathrm{MMIC}$ 의 두 서브스트레이트 의 유전율이 다르기 때문에, 각각 유효 유전상수를 $\chi_{L}$ 과 $x_{M}$ 으로 표시한다.

$$
\begin{gathered}
C_{L}=\frac{\left(\sqrt{\varkappa_{L}} / Z_{i m} v_{0}\right) D_{L}}{2}(F) \\
C_{M}=\frac{\left(\sqrt{\varkappa_{M}} / Z_{i m} v_{0}\right) D_{L}}{2}(F)
\end{gathered}
$$

$\mathrm{Ka}$-대역에서의 단일 본딩 와이어의 자체 인덕턴스, 저 항 및 커패시턴스 성분을 식 (1) 식 (4)를 활용하여 계산 및 분석할 수 있다. 제안된 단일 본딩 와이어의 파라미터 를 표 1에 정리하였다.

$\mathrm{Ka}$-대역의 등가회로 모델을 그림 4와 같이 제시하였 다. $i$ 번째 와이어의 인덕턴스 성분을 $L_{w i}$ 로 표시한다. 와 이어의 개수가 증가함에 따라 와이어 간의 상호 인덕턴 스 $(M)$ 와 커플링 계수를 고려하여 $D_{L} / d_{s} \gg 1$ 일 때에 식 (5)와 같이 나타낼 수 있다 ${ }^{[10]} . i$ 번째 와이어와 $j$ 번째 와이 어 간의 커플링 계수 $\left(k_{i j}\right)$ 로 표시하여 $i, j=1,2,3$ 으로 제 시하였다. 자기 상수는 $\mu_{0}$ 로 표시하고, 와이어간 중심 거 리는 $d_{s}$ 로 제시하였다.

$$
M \simeq \frac{\mu_{0} D_{L}}{2 \pi}\left[\log _{e}\left(\frac{2 D_{L}}{d_{s}}-1+\frac{d_{s}}{D_{L}}\right)\right]
$$

표 1. 단일 본딩 와이어의 파라미터

Table 1. Parameters of single bonding wire.

\begin{tabular}{|c|c|c|c|c|c|}
\hline \multicolumn{6}{|c|}{ Parameter of single bonding wire } \\
\hline$H_{1}$ & $0.1 \mathrm{~mm}$ & $L_{w}$ & $0.24 \mathrm{nH}$ & $Z_{\text {im }}$ & $165 \Omega$ \\
\hline$D_{1}$ & $0.18 \mathrm{~mm}$ & $R_{w}$ & $0.021 \Omega$ & $x_{\text {eff }}$ & $1 \mathrm{fF}$ \\
\hline$D_{2}$ & $0.158 \mathrm{~mm}$ & $C_{L}$ & $1.94 \mathrm{fF}$ & \multirow{2}{*}{$\sigma$} & $\begin{array}{c}4.1 \times 10^{7} \Omega / \mathrm{m} \\
(\text { Gold wire })\end{array}$ \\
\hline$d_{p}$ & $0.3 \mathrm{~mm}$ & $C_{M}$ & $1.87 \mathrm{fF}$ & & \multirow{2}{*}{$5 \times 10^{-4} \mathrm{~m}^{2}$} \\
\cline { 1 - 4 }$a$ & $0.0254 \mathrm{~mm}$ & $D_{L}$ & $0.438 \mathrm{~mm}$ & \multirow{2}{*}{ A } & \\
\cline { 1 - 4 } & 8.6 & $\varepsilon_{M}$ & 3.5 & &
\end{tabular}




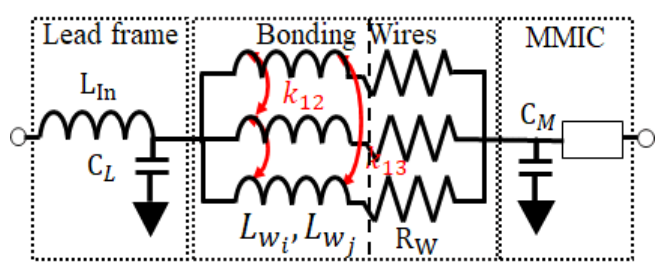

그림 4. $29 \mathrm{GHz}$ 에서의 본딩 와이어 등가회로 모델

Fig. 4. Equivalent circuit model of bonding wire at $29 \mathrm{GHz}$.

$$
k_{i j}=\frac{M}{\sqrt{L_{w_{i}} L_{w_{j}}}}
$$

그림 4에서 패키지의 Lead-frame Via에서 발생하는 인 덕턴스를 $L_{I n}$ 으로 제시하고, 본딩 와이어와 Lead-frame 사이에서 발생한 커패시턴스를 $C_{L}, \mathrm{MMIC}$ 사이에서 발생 한 커패시턴스를 $C_{M}$ 으로 표시하였다.

제안된 본딩 와이어의 길이 $\left(D_{L}\right)$ 는 $0.438 \mathrm{~mm}$ 이고, 개 수가 3 개인 경우에 대해 도출한 본딩 와이어의 성분들을 단일 본딩 와이어에 적용하여 파라미터 값들을 얻었다. $C_{L}$ 이 $35.26 f F, L_{w}$ 는 $0.29 n H, R_{w}$ 는 $0.0215 \Omega, C_{M}$ 은 $119.6 f F$ 이고, 와이어의 간에 커플링 계수인 $k_{12}$ 는 0.92 , $k_{13}$ 은 0.72 로 확인되었다. $29 \mathrm{GHz}$ 에서의 본딩 와이어 등 가회로의 파라미터를 표 2에 도시하였다.

\section{III. 본딩 와이어 시뮬레이션 및 분석}

7×7 mm 24-pin QFN 패키지의 3D 모델은 그림 5(a)와 같다. 그림 5(b)에 제시된 패키지의 파라미터와 같이 패키 지의 신호 Lead-frame 폭은 $0.22 \mathrm{~mm}$ 이고, 양쪽 간격은 $0.77 \mathrm{~mm}$ 이다. 그림 $5(\mathrm{c})$ 는 $\mathrm{QFN}$ 패키지의 단면도로, 본딩 와이어는 MMIC와 신호 Lead-frame을 연결한다.

그림 6(a)와 같이, QFN 패키지의 Lead-frame to bonding

표 2. 본딩 와이어 등가회로의 파라미터

Table 2. Parameters of bonding wire equivalent circuit.

\begin{tabular}{|c|c|c|c|c|}
\hline \multicolumn{5}{|c|}{ Parameter of bonding wire circuit model @29 $\mathrm{GHz}$} \\
\hline$L_{I n}$ & $C_{L}$ & $L_{w}$ & $R_{w}$ & $C_{M}$ \\
\hline $0.455 \mathrm{nH}$ & $35.26 \mathrm{nH}$ & $0.29 \mathrm{nH}$ & $0.0215 \Omega$ & $119.6 \mathrm{nH}$ \\
\hline \multicolumn{2}{|c|}{$k_{12}$} & \multicolumn{2}{|c|}{$k_{13}$} & \multicolumn{2}{|c|}{$d_{s}$} \\
\hline 0.92 & 0.72 & \multicolumn{2}{|c|}{$0.05 \mathrm{~mm}$} \\
\hline
\end{tabular}

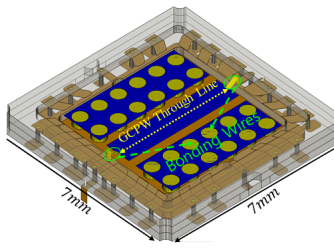

(a) $3 \mathrm{D}$ 모델

(a) 3D model

\begin{tabular}{|c|c|}
\hline Size & $7 \times 7 \mathrm{~mm}$ \\
\hline Lead width & $0.22 \mathrm{~mm}$ \\
\hline Total thickness & $0.75 \mathrm{~mm}$ \\
\hline Lead pitch & $0.77 \mathrm{~mm}$ \\
\hline Pin count & 24 \\
\hline
\end{tabular}

(b) QFN 패키지 파라미터 테이블 (b) Parameter table of QFN package

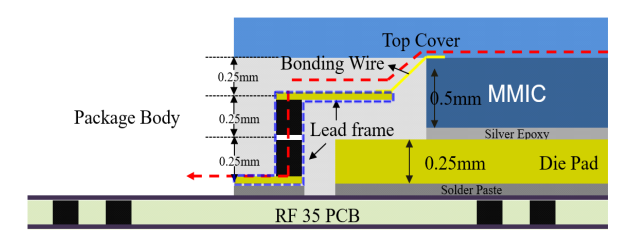

(c) QFN 패키지의 단면도

(c) Cross-section view of QFN package

그림 5. 제안하는 $\mathrm{QFN}$ 패키지의 3차원 구조

Fig. 5. 3D structure of the proposed QFN package.

wire to MMIC EM 3D 모델에서 와이어의 개수 $\left(N_{k}\right)$ 및 길 이 $\left(D_{L}\right)$ 는 등가회로 모델과 같은 값으로 설계되었다. Ka대역에서의 $\mathrm{QFN}$ 패키지 본딩 와이어 특성의 검증을 위 해 MMIC 칩을 GCPW(ground coplanar wave guide) through 선로로 대체하였고, 설계된 QFN 패키지의 측면 도를 그림 $6(\mathrm{~b})$ 에서 제시하였다. 표 2에서 설계한 본딩 와 이어가 3 개인 등가회로 모델의 모의실험과 그림 6 에서 Cadence 사의 AWR EM 시뮬레이션 결과를 그림 7과 같 이 비교하였다.

그림 6(a)에서 와이어의 길이 $\left(D_{L}\right)$ 를 $438 \mu \mathrm{m}$ 로 고정시 키고, 본딩 와이어의 개수를 1 개부터 8 개까지 1 개씩 증가 시키면서 진행한 $\mathrm{EM}$ 시뮬레이션의 $S$-파라미터 결과를 그림 8 에 도시하였다.

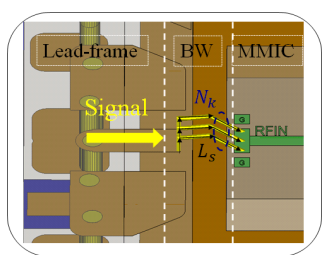

(a) 평면도

(a) Top view

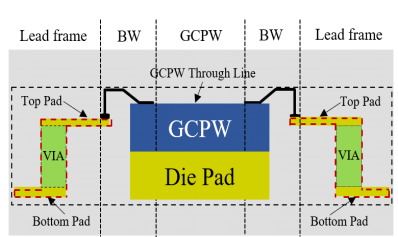

(b) 측면도

(b) Side view
그림 6. 제안하는 $\mathrm{QFN}$ 패키지의 형태

Fig. 6. Configuration of the proposed QFN package. 


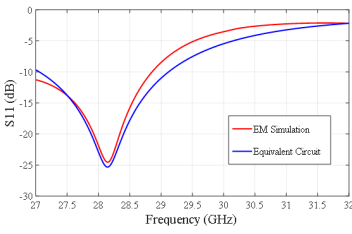

(a) $S_{11}$ (반사 손실)

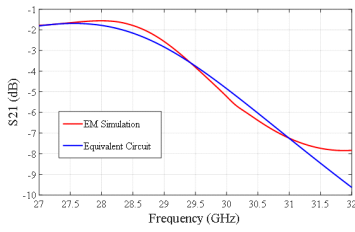

(b) $S_{21}$ (삽입 손실)

(b) $S_{21}$ (insertion loss)
그림 7. 등가회로 모델과 AWR EM 시뮬레이션 결과 분석

Fig. 7. Analysis of equivalent circuit model and AWR EM simulation.

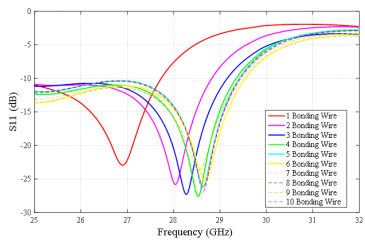

(a) $S_{11}$ (반사 손실)

(a) $S_{11}$ (returen loss)

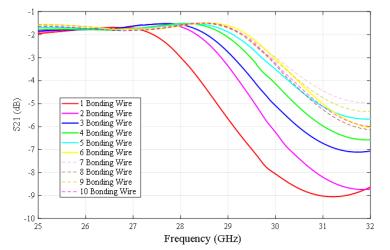

(b) $S_{21}$ (삽입 손실)

(b) $S_{21}$ (insertion loss)
그림 8. 본딩 와이어의 개수에 따른 $\mathrm{EM}$ 시뮬레이션

Fig. 8. EM simulation by number of bonding wire.

그림 8 과 같이 $S_{11}$ 의 공진 주파수는 와이어의 개수를 1 개부터 6 개까지 증가시킴에 따라 $0.9 \mathrm{GHz}, 0.1 \mathrm{GHz}, 0.37$ $\mathrm{GHz}, 0.15 \mathrm{GHz}, 0.49 \mathrm{GHz}$ 만큼 증가하였고, 6개부터 8개까 지 증가시킴에 따라 와이어 간의 과도결합으로 인해 0.33 $\mathrm{GHz}, 0.07 \mathrm{GHz}$ 만큼 감소하였다. 와이어 개수에 따른 시 뮬레이션 결과를 표 3 과 같이 제시하였다.

표 3. 와이어 개수에 따른 $\mathrm{QFN}$ 패키지 시뮬레이션 결과 Table 3. Simulation result of the QFN package by the number of bonding wire.

\begin{tabular}{|c|c|c|c|c|}
\hline \multicolumn{5}{|c|}{ Ka-band QFN package simulation of wire quantity } \\
\hline$N_{k}$ & $D_{L}$ & $S_{11}$ & $S_{21}$ & Resonance frequency \\
\hline 1 & \multirow{8}{*}{$\begin{array}{l}438 \\
\mu \mathrm{m}\end{array}$} & $-3.3 \mathrm{~dB}$ & $-5.6 \mathrm{~dB}$ & $26.9 \mathrm{GHz}$ \\
\hline 2 & & $-6.1 \mathrm{~dB}$ & $-3.3 \mathrm{~dB}$ & $27.7 \mathrm{GHz}$ \\
\hline 3 & & $-7.6 \mathrm{~dB}$ & $-2.7 \mathrm{~dB}$ & $27.9 \mathrm{GHz}$ \\
\hline 4 & & $-10.8 \mathrm{~dB}$ & $-2.1 \mathrm{~dB}$ & $28.6 \mathrm{GHz}$ \\
\hline 5 & & $-13.5 \mathrm{~dB}$ & $-1.8 \mathrm{~dB}$ & $28.4 \mathrm{GHz}$ \\
\hline 6 & & $-22.0 \mathrm{~dB}$ & $-1.5 \mathrm{~dB}$ & $28.8 \mathrm{GHz}$ \\
\hline 7 & & $-16.4 \mathrm{~dB}$ & $-1.7 \mathrm{~dB}$ & $28.5 \mathrm{GHz}$ \\
\hline 8 & & $-16.5 \mathrm{~dB}$ & $-1.7 \mathrm{~dB}$ & $28.6 \mathrm{GHz}$ \\
\hline
\end{tabular}

위의 시뮬레이션 결과를 바탕으로 와이어의 개수를 6 개로 설계하고 와이어의 길이 $\left(D_{L}\right)$ 를 변화시키면서 진행 한 시뮬레이션의 결과를 그림 9에 제시하였다. 와이어의 길이가 $238 \mu \mathrm{m}, 48 \mu \mathrm{m}$ 만큼 감소함에 따라, 와이어의 병렬 저항과 자체 인덕턴스 성분이 감소하여 공진 주파 수가 $1.75 \mathrm{GHz}, 0.95 \mathrm{GHz}$ 만큼 변화하였다. 와이어 길이 에 따른 $\mathrm{QFN}$ 패키지 시뮬레이션 결과를 표 4에 정리했 다. 와이어 길이와 개수에 따른 시뮬레이션의 스미스 차 트 결과는 그림 10 에서 제시하였다.

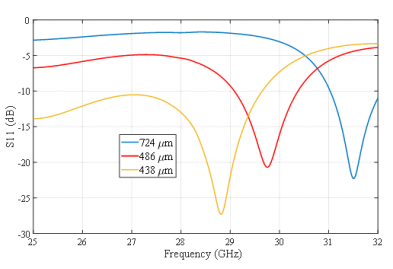

(a) $S_{11}$ (반사 손실)

(a) $S_{11}$ (returen loss)

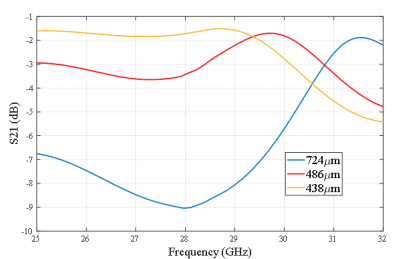

(b) $S_{21}$ (삽입 손실)

(b) $S_{21}$ (insertion loss)
그림 9. 본딩 와이어의 길이에 따른 $\mathrm{EM}$ 시뮬레이션

Fig. 9. EM simulation by length of bonding wire.

표 4. 와이어 길이에 따른 $\mathrm{QFN}$ 패키지 시뮬레이션 결과 Table 4. QFN package simulation result of wire length.

\begin{tabular}{|c|c|c|c|c|}
\hline \multicolumn{5}{|c|}{ Ka-band QFN package simulation of wire length } \\
\hline \multirow{2}{*}{$N_{k}$} & $D_{L}$ & $S_{11}$ & $S_{21}$ & Resonance frequency \\
\hline \multirow{3}{*}{6} & $724 \mu \mathrm{m}$ & $-1.9 \mathrm{~dB}$ & $-8.1 \mathrm{~dB}$ & $31.5 \mathrm{GHz}$ \\
\cline { 2 - 5 } & $486 \mu \mathrm{m}$ & $-9.2 \mathrm{~dB}$ & $-2.2 \mathrm{~dB}$ & $29.7 \mathrm{GHz}$ \\
\cline { 2 - 5 } & $438 \mu \mathrm{m}$ & $-22 \mathrm{~dB}$ & $-1.5 \mathrm{~dB}$ & $28.8 \mathrm{GHz}$ \\
\hline
\end{tabular}

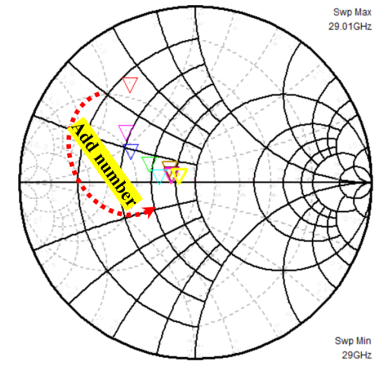

(a) 개수 증가

(a) Add number

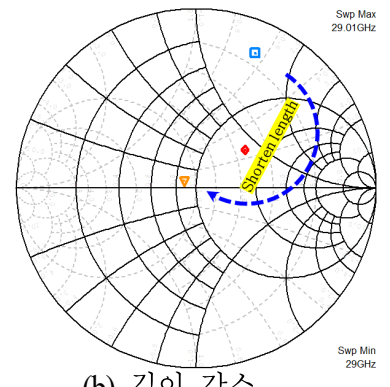

(b) 길이 감소

(b) Shorten length
그림 10. 본딩 와이어의 $\mathrm{EM}$ 스미스 차트 결과

Fig. 10. EM smith chart result of bonding wire. 
THE JOURNAL OF KOREAN INSTITUTE OF ELECTROMAGNETIC ENGINEERING AND SCIENCE. vol. 32, no. 11, November. 2021.

\section{Ka-대역 QFN 패키지 본딩 와이어의 설계 및 실험}

다층 $\mathrm{HTCC}$ (high temperature co-fired ceramic) 공정으로 제작된 24-pin $7 \times 7 \mathrm{~mm}$ QFN 패키지를 그림 11(a)와 같이 제시하였다. 디자인된 $\mathrm{QFN}$ 패키지의 신호 패드를 와이어 본딩 방식으로 연결한 구조는 그림 11(b)와 같다.

$\mathrm{QFN}$ 패키지 본딩 와이어의 특성을 분석하기 위해 그 림 12 와 같은 측정 환경을 구성하여 $\mathrm{GCPW}$ 선로를 설계 하였고, MMIC를 GCPW through 선로로 대체하였다. S-파 라미터를 분석하기 위해 Keysight 사의 P5007A 벡터 네트 워크 분석기를 사용하였다.

$\mathrm{RF} 35$ Main Board를 바탕으로 한 Ka-대역 QFN 패키지 에서 길이 $\left(D_{L}\right)$ 는 $426 \mu \mathrm{m}$ 로 고정하고, 개수 $\left(N_{k}\right)$ 를 3,6 , 10 으로 변화시키며 실험을 진행하였다. 그림 13 은 실측과 등가회로 모델링한 QFN 패키지의 $S_{11}$ (반사 손실), $S_{21}$ (삽 입 손실)에 대한 그래프이다. 본딩 와이어의 개수를 3 개 에서 6개로 변화할 때는 패키지의 공진 주파수가 증가하 지만, 10 개 경우는 공진 주파수가 감소하였다. 따라서 본 딩 와이어의 개수가 6 개 이상이면, 와이어 간의 과도결합 이 발생하여 공진 주파수가 감소하고, 삽입 손실이 증가 한 것을 등가회로 모델 결과를 통해서 검증할 수 있다.

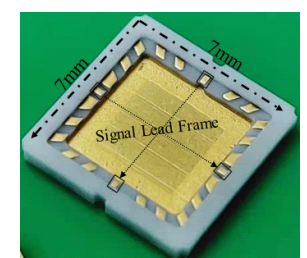

(a) $7 \times 7 \mathrm{~mm}$ QFN 패키지

(a) $7 \times 7 \mathrm{~mm}$ QFN package

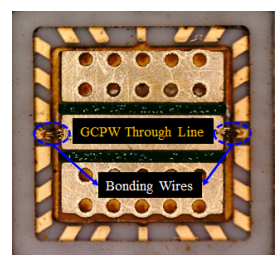

(b) GCPW through 선로

(b) GCPW through line
그림 11. QFN 패키지 및 GCPW through 선로의 구조

Fig. 11. Structure of QFN package and GCPW through line.
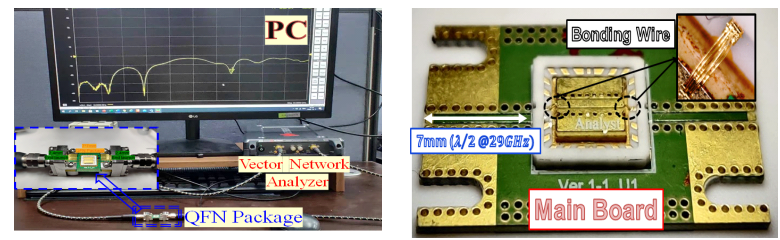

그림 12. $\mathrm{QFN}$ 패키지 샘플 및 측정 환경

Fig. 12. QFN package sample and measurement environment.

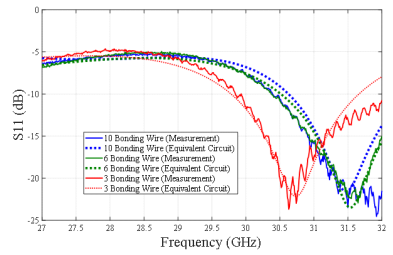

(a) $S_{11}$ (반사 손실)

(a) $S_{11}$ (returen loss)

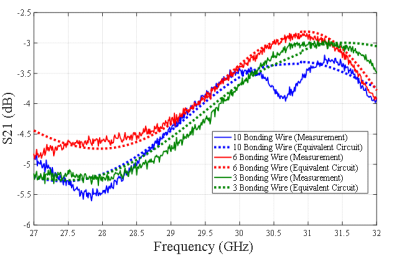

(b) $S_{21}$ (삽입 손실)

(b) $S_{21}$ (insertion loss)
그림 13. $N_{k}$ 에 따른 측정과 등가회로 모델 비교

Fig. 13. Comparison of measurement and equivalent circuit model result of different $N_{k}$.

표 5에서의 측정 결과를 기반으로 $N_{k}$ 가 6개일 때의 본 딩 와이어 길이에 따른 실험을 진행하였다. Ka-대역 $\mathrm{QFN}$ 패키지 본딩 와이어의 $29 \mathrm{GHz}$ 에서 $\mathrm{RF}$ 특성을 검증하기 위해 제안된 본딩 와이어 길이는 GCPW through 선로 길 이에 따라 결정됨으로써 선로 길이를 $3.5 \mathrm{~mm}, 4.14 \mathrm{~mm}$, $4.32 \mathrm{~mm}$ 로 설계 및 제작하였다.

그림 14 에서 본딩 와이어 길이는 패키지의 정합 위치 및 삽입 손실에 영향을 미치는 것을 볼 수 있다. 본딩 와 이어의 길이는 $\mathrm{GCPW}$ through 선로의 길이가 증가함에 따라 감소한다. 따라서, 와이어의 길이를 $438 \mu \mathrm{m}, 486 \mu \mathrm{m}$,

표 5. $29 \mathrm{GHz}$ 에서 $N_{k}$ 에 따른 측정 결과

Table 5. Measurement result of different $N_{k}$ at $29 \mathrm{GHz}$.

\begin{tabular}{|c|c|c|c|c|}
\hline & $N_{k}$ & $D_{L}$ & $S_{11}$ & $S_{21}$ \\
\cline { 2 - 3 } Ka-band & 3 & & $-5.8 \mathrm{~dB}$ & $-4.4 \mathrm{~dB}$ \\
\cline { 2 - 3 } @29 GHz & 6 & 426 & $-5.4 \mathrm{~dB}$ & $-4.7 \mathrm{~dB}$ \\
\cline { 2 - 3 } \cline { 4 - 5 } & 10 & $\mu \mathrm{m}$ & $-5.2 \mathrm{~dB}$ & $-4.3 \mathrm{~dB}$ \\
\hline
\end{tabular}

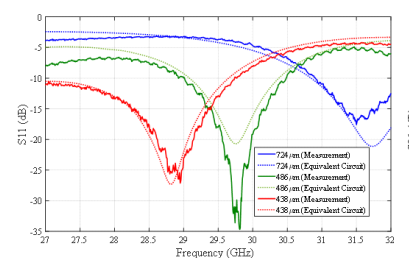

(a) $S_{11}$ (반사 손실)

(a) $S_{11}$ (returen loss)

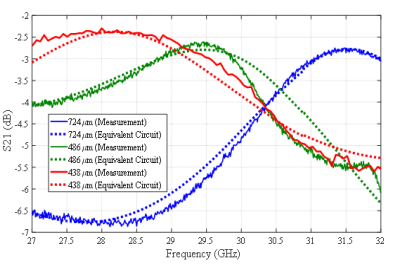

(b) $S_{21}$ (삽입 손실)
그림 14. 본딩 와이어 길이 변화에 따른 실측과 등가회 로 결과 비교

Fig. 14. Comparison of measurement and equivalent circuit result to different lengths. 
표 6. $29 \mathrm{GHz}$ 에서 $D_{L}$ 에 따른 측정 결과

Table 6. Measurement result of different $D_{L}$ at $29 \mathrm{GHz}$.

\begin{tabular}{|c|c|c|c|c|}
\hline \multirow{4}{*}{$\begin{array}{l}\text { Ka-band } \\
\text { @ } 29 \mathrm{GHz}\end{array}$} & $N_{k}$ & $D_{L}$ & $S_{11}$ & $S_{21}$ \\
\hline & \multirow{3}{*}{6} & $438 \mu \mathrm{m}$ & $-24.8 \mathrm{~dB}$ & $-1.8 \mathrm{~dB}$ \\
\hline & & $486 \mu \mathrm{m}$ & $-10.4 \mathrm{~dB}$ & $-3.0 \mathrm{~dB}$ \\
\hline & & $724 \mu \mathrm{m}$ & $-8.9 \mathrm{~dB}$ & $-3.5 \mathrm{~dB}$ \\
\hline
\end{tabular}

$724 \mu \mathrm{m}$ 로 설계하여 제작 및 실측하였다.

$\mathrm{QFN}$ 패키지의 $S$-파라미터는 표 6과 같이 본딩 와이어 길이가 $438 \mu \mathrm{m}$ 일 때, $29 \mathrm{GHz}$ 대역에서 $S_{11}$ 가 $-26 \mathrm{~dB}$, $S_{21}$ 이 $-1.8 \mathrm{~dB}$ 로 실측된다. 와이어 길이가 짧아지면서 자체 인덕턴스 성분 및 기생 저항 성분이 작아지므로 삽 입 손실이 $1 \mathrm{~dB}$ 개선됨을 확인하였다.

\section{$\mathrm{V}$. 결 론}

본 논문에서는 GCPW through 선로와 $\mathrm{QFN}$ 패키지 Lead-frame 간의 다중 본딩 와이어 트랜지션의 방식에 대 해 $\mathrm{RF}$ 특성 검증 기법 및 패키지 $3 \mathrm{D} \mathrm{EM}$ 시뮬레이션 과 정을 제시하였다.

$\mathrm{Ka}-$ 대역 본딩 와이어의 등가 모델링을 제시하고, 와이 어의 개수 및 길이 변화에 따른 특성을 모의실험과 실측 결과를 통해 검증하였다. $438 \mu \mathrm{m}$ 인 단일 및 다중 본딩 와이어의 기생 성분을 기하학적 접근법과 등가회로 모델 을 통해서 도출하였고, $\mathrm{EM}$ 시뮬레이션 결과와 비교하여 확인하였다. 본 연구는 $5 \mathrm{G}$ 용 $\mathrm{QFN}$ 패키지에서 $\mathrm{MMIC}$ 와 패키지의 본딩 와이어 연결 구조 설계 및 특성을 분석에 활용될 수 있을 것으로 기대된다.

\section{References}

[1] J. Lu, H. Jia, A. Arias, X. Gong, and Z. J. Shen "Onchip bond wire transformers for power SOC applications," in 2008 Twenty-Third Annual IEEE Applied Power Electronics Conference and Exposition, Austin, TX, Mar. 2008, vol. 8, no. 7, pp. 199-204.

[2] M. J. Chen, S. A. Tabatabaei, "Boardband, thin-film, liquid crystal polymer air-cavity quad flat no-lead(QFN) package," in 2009 Annual IEEE Compound Semiconductor
Integrated Circuit Symposium, Greensboro, NC, Oct. 2009, pp. 1-4.

[3] C. C. Wei, C. T. Fan, and T. H. Chiang, "A comparison study of high-frequency performance between ball bonding and ribbon bonding," in 2009 4th International Microsystems, Packaging, Assembly and Circuits Technology Conference, Taipei, Oct. 2009, pp. 685-688.

[4] C. H. Chen, Y. C. Lin, Y. T. Shih, S. C. Chen, C. H. Tsai, and S. C. Wang, et al., "Evaluation of corrosion resistance of ag-alloy bonding wires for electronic packaging," IEEE Transactions on Components, Packaging and Manufacturing Technology, vol. 8, no. 1, pp. 146153, Jan. 2018.

[5] T. P. Wang, Y. F Lu, "Fast and accurate frequencydependent behavioral model of bonding wires," IEEE Transactions on Industrial Informatics, vol. 13, no. 5, pp. 2389-2396, Oct. 2017.

[6] W. Tian, H. Cui, and W. Yu, "Analysis and experimental test of electrical characteristics on bonding wire," Electronics 2019, vol. 8, no. 3, p. 365, Mar. 2019.

[7] J. H. Lim, D. H. Kwon, and J. S. Rieh, "RF characterization and modeling of various wire bond transitions," IEEE Transactions on Advance Packaging, vol. 28, no. 4, pp. 772-778, Nov. 2005.

[8] Y. C. Lin, W. H. Lee, T. S. Horng, and L. T. Hwang, "Full chip-package-board co-design of broadband QFN bonding transition using backside via and defected ground structure," IEEE Transactions on Components, Packaging and Manufacturing Technology, vol. 4, no. 9, pp. 1470-1479, Sep. 2014.

[9] R. Sturdivant, CTO, "Broadband electrical modeling of transitions and interconnects useful for PCB and co-fired ceramic packaging," in 2014 IMAPS RaMP Conference, San Diego, CA, Apr. 2014.

[10] X. N. Qi, "High frequency characterization and modeling of on interconnects and RF IC wire bonds," Ph.D. dissertation, Stanford University, Stanford, CA, 2001. 
THE JOURNAL OF KOREAN INSTITUTE OF ELECTROMAGNETIC ENGINEERING AND SCIENCE. vol. 32, no. 11, November. 2021.

\section{왕 문 걸 [건국대학교/석사과정]}

$$
\text { https://orcid.org/0000-0002-7310-0294 }
$$

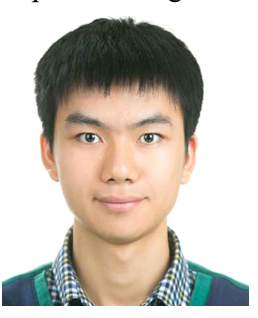

2020년 2월: 건국대학교 전자공학과 (공학 사)

2020년 3월 현재: 건국대학교 전자정보 통신공학과 석사과정

[주 관심분야] Semiconductor Packaging, Wireless Power Transfer 등

$$
\text { 구 현 철 [건국대학교/교수] }
$$

$$
\text { https://orcid.org/0000-0002-1961-5166 }
$$

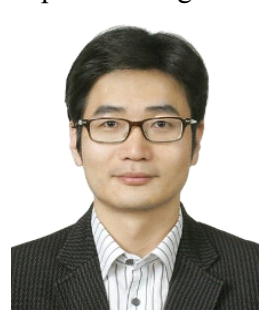

1995년 2월: 서울대학교 전기공학과 (공학

1997년 2월: 서울대학교 전기공학과 (공학 석사)

2003년 12월: 미국 Georgia Institute of Technology University 전기전자 및 컴퓨터 공학과 (공학박사)

2005년 3월 현재: 건국대학교 전기전자공학부 교수

[주 관심분야] RF Power Amplifier, Digital RF, RF Front-End Design, Wireless Power Transfer System 등
강 원 실 [알에프머트리얼즈(주)/수석연구원]

https://orcid.org/0000-0002-3428-9733

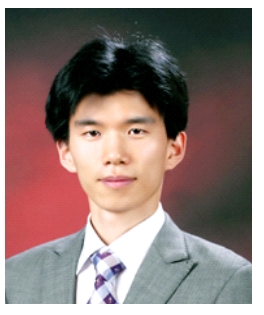

2008년 2월: 건국대학교 전자공학과 (공학 사)

2018년 8월: 건국대학교 전자정보통신공 학과 (공학박사)

2018년 9월: 차세대무선전원센터 책임연 구원, 건국대학교 강의 초빙교수

2020년 8월 현재: 알에프머트리얼즈 (주)

연구소 수석연구원

[주 관심분야] RF Power Amplifier, HTCC Package, Digital RF, Digital Radar System, Multi-Array Beamforming, RF Wireless Power Transfer 등 